\title{
humanidades
}

Revista humanidades

Diciembre, 2014 •Volumen 4 •ISSN 2215-3934・pp. 1-29

\section{Los Hebreos: Un legado religioso}

DOI: http://dx.doi.org/10.15517/h.v4i1.16320

Recibido: 23-Abril-2014 / Aceptado: 20-Junio-2014

\section{Ligia Carvajal Mena}

Doctora, profesora Catedrática en la Sección de Historia de la Cultura en la Escuela de Estudios Generales de la Universidad de Costa Rica.

Correo electrónico: carvajalligia@ hotmail.com 


\section{Los Hebreos: Un legado religioso}

\section{Resumen}

Los primeros asentamientos humanos tuvieron lugar en las riberas de ríos como el Éufrates, Tigris y Nilo. Estas tierras, por su fertilidad, se convirtieron en cuna de culturas que dejaron un legado importante para el desarrollo de la humanidad.

En el marco de esas culturas destaca la hebrea que asume aspectos culturales foráneos, los recompone y da un salto original en su cultura, especialmente en el ámbito religioso. Lo anterior al establecer inequívocamente el monoteísmo, realizar mejores construcciones (entre lo religioso y lo astronómico) y crear un Dios con fuerte contenido ético, que trasciende del ámbito nacional al universal.

\section{The Hebrews: A religious legacy}

\section{Abstract}

The first human settlements took place in the river banks such as the Euphrates, Tigris and Nile. Because of their fertility, those lands became the cradle of cultures that left an important legacy for the development of humanity.

Within the framework of these cultures is the Hebrew that assuming foreign cultural aspects, reassembled them and made an original break to their culture, especially in the religious area by establishing unequivocally the monotheism, also outstanding buildings combining the religious with the astronomical, as well as the creation of a God with strong ethical background, transcending the national scope to an universal approach.
Palabras clave: Cultura, religión, astronomía, calendarios, nomadismo, monoteísmo.
Keywords: Culture, religion, astronomy, calendars, nomadism, monotheism. 
Existen espacios geográficos que, por sus características, sirvieron de cuna a pueblos primitivos $\mathrm{y}$, con el transcurrir del tiempo, permitieron el desarrollo de culturas antiguas, tal es el caso de las tierras comprendidas entre los ríos Éufrates y Tigris (conocida como Mesopotamia) y aquellas irrigadas por el río Nilo, en Egipto. Dichas áreas se convirtieron en focos de desarrollo político y cultural, en ese sentido, dejaron una huella en la historia de la humanidad. Lo anterior porque allí se establecieron los primeros grandes Estados, durante el principio del tercer milenio, quizás por las particularidades específicas de estas zonas que favorecieron el desarrollo de una economía estable.

Las tierras mesopotámicas vieron nacer culturas que conformaron destacados imperios como la sumeria que avanzó significativamente en la agricultura, la escritura, el uso de metales como el bronce y el cobre, comprendió los movimientos de las estrellas, perfeccionó una fe y un sistema de prácticas religiosas normadas por un sacerdocio.

Se puede mencionar, además, el Imperio Babilónico. Esta potencia sostuvo guerras con los asirios y egipcios. En el ámbito cultural, destacó por su arquitectura, con la redificación de templos antiguos y la construcción de un palacio imperial muy grande que tenía terrazas y jardines colgantes, el cual, según los griegos, constituía una de las siete maravillas del mundo.

\section{Establecimiento de las primeras civilizaciones}


La cultura Babilónica, asimismo, legó la codificación de Hammurabi (creada en 1760 antes de Cristo) que es considerada la primera legislación de la historia. Este ordenamiento fue plasmado en una estela de piedra y se caracteriza por ser un documento que contemplaba la ley común de la época. Fue ubicado en el templo del dios babilónico Marduk.

En el campo religioso, según Tunner (1974), ambas culturas contaban con millares de divinidades. Entre las más importantes: el dios Anú, dios del cielo; Enlil, dios del aire y del viento; Ea o Enki, dios de la tierra y de las aguas subterráneas frescas; Sin, el dios de la luna; Nintud, diosa de la fecundidad; y Utu, dios del Sol. Babilonia heredó todo un sistema religioso; sumerios y babilonios crearon las bases para el desarrollo de la ciencia, lo que podría denominarse una protociencia.

La cultura Asiria, por su parte, asentada en la parte superior del río Tigris, se caracterizó por su dedicación al comercio, la minería y la innovación en el arte. Logró dominar la región media del Éufrates, la de Gosen, en Egipto, y conquistó todo el territorio de la Mesopotamia.

Otro legado cultural importante fue el de los Hicsos, quienes se apoderaron del control de Egipto y adoptaron al dios local Re o Ra, o dios Sol, que fue 
plasmado en el arte como un hombre halcón. Fueron monoteístas y dos de sus principales representantes fueron Akenatón y Nefertiti.

Ahora bien, la historia señala la existencia de estas tierras; sin embargo, aparece, de igual forma, el desierto como escenario geográfico. Se trata de una suerte de punto de partida de diversos grupos humanos, quienes buscaban mejores condiciones, como las que ofrecía la Mesopotamia y el Nilo, es decir, las tierras fértiles.

En el marco de la dinámica migratoria, algunos pueblos, como los amorreos, decidieron arraigarse en la Mesopotamia occidental y en Palestina. Otros grupos optaron por desplazarse a Siria y a la Mesopotamia septentrional; se convirtieron, entonces, en los antecesores de los arameos, mencionados en el texto bíblico. En dicho texto, además, se mencionan los edomitas, los moabitas y otras tribus que se ubicaron al este del mar Muerto, en las extensiones de Palestina, que tenían una situación geográfica estratégica importante.

Lo anterior les permitió tener influencia egipcia y babilónica, a tal punto que en el tercer milenio pasaron a formar parte del Imperio Babilónico y reconocieron la autoridad del Rey Hammurabi. De esa forma, incorporaron a su cultura la lengua, la escritura, las costumbres, las leyes, así como los mitos babilónicos. 
Así las cosas, los hebreos entraron a Palestina mediante dos procesos migratorios. El primero, con los amorreos a finales del siglo XVIII antes de Cristo y, el segundo, en los siglos XIV y XIII antes de Cristo, durante la desorganización del Imperio Egipcio. Cabe señalar que alrededor de 1750 antes de Cristo, las principales ciudades de Siria y Babilonia eran gobernadas por dinastías amorreas.

Varias tribus hebreas emigraron a Egipto y permanecieron en ese territorio alrededor de cuatrocientos años. De hecho, los hebreos instaurados en Egipto experimentaron la opresión y fueron dirigidos por Moisés; emprendieron su éxodo en el año 1165 antes de Cristo. Este líder, Moisés, los condujo a la península de Sinaí y los persuadió para que ellos, y otros hebreos que vivían en los contornos de Cades, se convirtieran en admiradores de Yahweh (el dios de los pastores del Sinaí). Posteriormente, el mismo Moisés les asignó un ritual y les entregó un código; esta asociación constituyó el núcleo de parte de la nación hebrea, que se denominó Israel. Cuando Moisés reorganizó esas tribus, se dio un avance paulatino hacia el norte, entre el Mar Muerto y la llanura costeña.

Se debe recordar que Moisés nació, según las narraciones bíblicas, en los años en que el Faraón había ordenado que todo hijo de padres hebreos fuera condenado a la esclavitud. Este personaje, luego de ser abandonado por su madre, fue rescatado por la hija del Faraón, quien lo adoptó y lo educó de acuerdo con las normas y lujos del palacio. Sin embargo, Moisés, conocedor 
de su origen, no se olvidó de su pueblo y un día, al ver que maltrataban a un hebreo, mató a uno de los verdugos. Por lo anterior, tuvo que refugiarse en el desierto, donde conoció a Yahvé, y sintió la necesidad de salvar a su pueblo. De esa manera, se convirtió en el protagonista del Éxodo, en constructor de la nación y fundador de la religión hebrea, pues los condujo a Canaán y les enseñó la existencia de un solo Dios.

Estas mismas tribus hebreas lograron conquistar las comarcas ubicadas al este del río Jordán y en su valle. Dominaron Jericó, en el siglo XII antes de Cristo, y, paulatinamente, tomaron Siquem, ciudad situada en la meseta. Allí, Josué dio a las tribus un código y una liturgia que, según se cree, fue copiada de los cananeos. Posteriormente, estas tribus se unieron y estructuraron la nación hebrea denominada Israel, que años más tarde, con la división del reino después de la muerte de Salomón, fue dominado - al norte- por diez de las tribus hebreas originales lideradas por la tribu de Efraín; estas siguieron llamándose Israel. Las tribus de Judá y de Benjamín -ubicadas en el sur- se autodenominaron con el nombre de Judea.

Los poderosos clanes de José, incluyendo los de Efraín, Manasés y Benjamín, conquistaron el territorio ubicado en el margen más lejano del río Jordán. Se establecieron allí algunos miembros del clan y avanzaron por el río. Después luchar por varios años, llegaron los clanes de Judá y sus asociados como Leví, Simeón y los kenitas, estos se introdujeron en la parte septentrional de 
Palestina, desde el desierto. A la luz de los estudiosos, el origen del pueblo hebreo se encuentra en el desierto de Arabia.

La conquista de Canaán resultó difícil para los hebreos y, antes de terminar este proceso, se vieron amenazados por los filisteos provenientes del Asia Menor y de las islas del mar Egeo, quienes los obligaron a ceder gran parte del territorio que habían conquistado. Pero, este hecho provocó la unificación hebrea y la fundación de la monarquía en el año 1025 antes de Cristo, porque hasta ese momento hombres sabios, llamados Jueces, fueron quienes gobernaron la nación (Mcnall, 1970, p. 119).

Los Jueces, llamados también caudillos carismáticos (porque se creía que tenían algún don especial por la gracia de Dios), fueron personajes apartados de sus clanes. Por su capacidad militar y de mando, sabiduría, así como la honestidad, se creía que estaban capacitados para solucionar disputas, ya sea a nivel familiar o entre individuos.

La constitución de los Jueces en el pueblo de Israel fue un rasgo distintivo entre este y otros pueblos que lo rodeaban, los cuales estaban altamente organizados; mientras que los hebreos eran una federación de tribus, unidas no por una figura política central, sino más bien, por una tradición común y un ligamen religioso o pacto, simbolizado en el "Arca del Pacto". 
De acuerdo con lo anterior, la monarquía hebrea se adoptó como una necesidad. Sin embargo, existió oposición a esta nueva forma de gobierno y circuló la voz de que el Dios de Israel era el verdadero Rey de su pueblo. En ese sentido, el primer rey hebreo fue Saúl (1028 a. C. - 1013 a. C.) descendiente de la tribu de Benjamín, el cual ocupaba ese cargo por una concesión de Dios. Es decir, el Rey se convirtió entonces, en un ungido de Dios. “[...] ¿no te ha ungido Yahvé por príncipe sobre su pueblo...?” (1 Samuel 10:1, La Biblia). Así pues, Saúl tenía la obligación de gobernar al pueblo de acuerdo con los dictados de la voluntad de Dios, según las revelaciones que hiciera a sus profetas. Saúl, a pesar de no tener un ejército permanente, logró rechazar a los filisteos y se propuso anexar las ciudades cananeas independientes. Este líder es considerado como la salvación política de Israel.

Al morirse Saúl, el reinado recayó en David (1013 a. C. - 973 a. C.) por un lapso de cuarenta años. En este tiempo aumentó la centralización del poder y David conformó un ejército con los leales al Rey, el cual derrocó por completo a los filisteos y unió a las doce tribus en un Estado fuerte, bajo un monarca absoluto. Inició, asimismo, la construcción de Jerusalén, sitio que nunca había sido conquistado por los hebreos. Esta ciudad, Jerusalén, se convirtió en el centro político del Estado por su posición estratégica entre los grupos del norte y del sur (2 Samuel 5:6, La Biblia). 
Un aspecto importante realizado por David fue el traslado del Arca del Pacto a Jerusalén y su protección. De esta manera, la corte llegó a ser no solo el centro de unidad política, sino también, el símbolo de la unidad religiosa.

Durante su reinado extendió su dominio a muchas tribus de las tierras altas y a varias ciudades situadas a lo largo del camino entre Damasco y Egipto. En ese sentido, hubo prosperidad material y aumento de la población; además, con la introducción del hierro al uso común, se produjo una revolución técnica que repercutió en la agricultura y en las construcciones. A este rey se le atribuye también la unión de la nación, el Estado y el culto.

El último rey de la monarquía que abarcó todo el territorio fue Salomón (973 a. C.- 933 a. C.) hijo de David. Aunque él no aumentó las conquistas hechas por su padre, se dedicó al desarrollo cultural y económico de su reino. Para este efecto, hizo alianzas políticas con países más pequeños y con Egipto y fundó nuevas ciudades. El establecimiento de la ruta histórica de Egipto a Mesopotamia, afirmó la monarquía hebrea, que ocupaba una posición importantísima en el comercio y en política internacional. Pero, su fortaleza era entendible por la debilidad de sus grandes vecinos: Babilonia, Asiria y Egipto.

Con el afán de impulsar su reino, Salomón incentivó la construcción, contó con el apoyo de Hiram y de Tiro, ciudades que formaban parte del Estado Fenicio. Este último Estado se especializaba en las artes industriales y 
comerciales, ya anteriormente el rey David había establecido relaciones amistosas selladas con una alianza durante el reinado de su hijo Salomón. Su anhelo era construir un templo digno de Yahvé y de sus sacerdotes; lo logró, pero, los gastos de la construcción recayeron directamente sobre el pueblo.

Sin embargo, por algún tiempo, los hebreos soñaron con que Jerusalén fuera una ciudad abierta -internacional- para ayudar al reino a tener el dominio mundial, tan aspirado por todos los Estados orientales. De hecho, los antiguos sentimientos de lealtad tribal se fusionaron, dando como resultado un nacionalismo intenso, pues entre las políticas del soberano se contemplaba el fortalecimiento y la consolidación de su propio territorio. Por este motivo, dividió el país en doce distritos, ignorando a propósito los límites tribales, con el fin de consolidar lo nacional, para lo cual, el Templo desempeñaba una función especial, para la realización de actividades religiosas.

Para unos, “[...] el templo se convirtió gradualmente en herencia de la nación, el centro de su vida religiosa [...] fue un símbolo de paz [...]" (León, 1943, p. 72); para otros, “[...] era ante todo una capilla del palacio [...] el esplendor del edificio debía servir para glorificar a la nación y a la dinastía [...]" (Lods, 1940, p. 90), porque por sus adornos y lujos, el templo tenía un parecido a los santuarios egipcios y fenicios, los cuales contrastaban con la mentalidad de los hebreos nómadas, que no requerían de lujo para comunicarse con su Dios. 
Pero, aunque Salomón fue considerado, según la tradición, como uno de los hombres más sabios, su gloria fue superficial, porque ese mismo oro, cuya acumulación pregonaba la prosperidad del país, provocó cambios económicos que intensificaron las contradicciones internas. Estas contradicciones constituían un peligro para la unidad nacional, pues la construcción del templo produjo un déficit en la economía de Palestina, que era pobre en recursos y por ello, Salomón

[...] cedió veinte ciudades y recurrió a la corvée, o sea, al trabajo oligatorio. Cada tres meses se reclutaban treinta mil hebreos y se los enviaba a Fenicia para que trabajasen en los bosques y minas del rey Hiram de Tiro, a quien se habían comprado los materiales más costosos [...] (McNalls, 1970, p.119).

Sumado a esto, el progreso de Asiria significaba también una amenaza para la nación.

Después de la muerte de Salomón, el descontento del pueblo creció, a tal punto que las diez tribus del norte no quisieron que su hijo Roboam los gobernara. Por eso, se separaron y establecieron su propio reino, denominado el Reino de Israel o Reino de Samaria. No obstante, en el año 722 a. C. fueron dominados por los asirios y “[...] estos reinos terminaron siendo absorbidos por las poblaciones más numerosas que los rodeaban. Desde entonces, se les llama las diez tribus perdidas de Israel [...]” (McNalls, 1970, p.119). 
Por su parte, las tribus del sur unidas fundaron Judea, como se señaló anteriormente. No obstante, en año 586 a. C., los caldeos los vencieron y Nabucodonosor destruyó totalmente Judá, Jerusalén y el Templo. Los habitantes de estas ciudades fueron esparcidos por el Imperio Babilónico, hasta que los caldeos cayeron bajo el dominio de Ciro el Persa, quien puso en libertad a los judíos y les permitió volver a su país de origen.

La memoria del pueblo hebreo se plasma en el texto bíblico, específicamente, en el Antiguo Testamento, que se encarga de retratar todas las vicisitudes que experimentó este pueblo. La historia de las tierras bíblicas, tanto antes como durante el período de Israel en Palestina, contienen muchos relatos de las sucesivas invasiones entre sí, de los reinos y de civilizaciones que se desarrollaron, pues la historia de la nación hebrea está impregnada de desplazamientos y duras luchas, como se apuntó anteriormente.

En el Antiguo Testamento se entrelazan inseparablemente la historia y la teología en un todo, dichas partes mantienen una conexión orgánica a lo largo de un desarrollo progresivo. "En él encontramos, una exposición coherente de la historia primitiva de Israel, una imagen que, no obstante sus grandes lagunas, nos impresiona por lo acabado del conjunto" (Martínez, 1987, p. 239). Además de la historia de Israel, se plasma la ideología de un pueblo que expone su fe. Esa amalgama de Historia y fe forman parte de una revelación otorgada al pueblo hebreo por medio de Moisés y los demás profetas. 
Ahora bien, los historiadores plantean que en la península de Arabia se establecieron los semitas o hijos del Sem, nómadas por naturaleza, con tradiciones pastoriles, cuya historia muestra una sociedad patriarcal en la que el padre constituía la cabeza de la familia y las mujeres estaban reducidas prácticamente a la servidumbre; ellas eran compradas o capturadas. La poligamia era una práctica común, los textos sagrados de los hebreos indican que Jacob y Salomón fueron polígamos. Estas características son esenciales para la conformación de la cultura hebrea.

Todas sociedades crean su propia cultura y la expresan por medio de diferentes manifestaciones. La cultura constituye, entonces, una muestra de pensamiento, de comunicación y de actuación de los seres humanos; la memoria de lo que ha sido y un registro imaginario de lo que pudo ser y hacer esa sociedad. Por eso, la cultura posibilita el conocimiento de los diversos procesos de construcción de sentido del desarrollo de la vida cotidiana y la realización de las prácticas culturales de la sociedad, las cuales están impregnadas de significación y abren espacio a la construcción de diversos discursos.

El desarrollo social de los hebreos fue el resultado de una acción recíproca de factores complejos y su cultura reúne materiales de muchas fuentes. La religión y el culto a Yahveh, que articuló las experiencias de las tribus en las batallas realizadas para dominar aquellos pueblos cuya cultura material era más avanzada, fue su elemento característico.

\section{Los hebreos y su cultura}


La religión hebrea evolucionó a lo largo del tiempo. Desde su aparición como pueblo nómada hasta la existencia de Moisés (1100a. C.), era una religión animista, que contaba con prácticas diversas de magia. Para ellos, casi todos los fenómenos ocurrían por la acción de personas o poderes sobrenaturales invisibles, parecidos al espíritu humano. De ahí que no existiera ninguna diferencia esencial entre lo espiritual y lo material; no obstante, sí admitían la existencia de una identidad práctica compuesta por cosas y acontecimientos. De hecho, la religión como expresión de la cultura, históricamente, se considera como un recurso que le permite a la persona satisfacer sus inquietudes sobre fenómenos del medio circundante. Además, es el campo de interacción del humano con el espacio y con la sociedad de la que forma parte. En este sentido, cabe mencionar que los hebreos veneraban las fuentes de agua, este líquido se consideraba dotado de conocimientos sobrenaturales, como se puede observar en el pronunciamiento de un juicio donde el agua santa era bebida por el infractor y se daba un veredicto pronunciando la frase "agua viva", que simbolizaba un espacio vital de interacción entre el desierto y las zonas vitalizadas por esta, como las riberas del río Jordán o manantiales naturales en diferentes lugares.

En el imaginario colectivo hebreo se contaba con numerosos dioses locales que no tenían nombre ni tradición y en torno a ellos. Según sus creencias, se agrupaban muchos espíritus malignos y benignos, entre estos, "Lilith" a quien, en algunas ocasiones, se le otorgaban características femeninas y, en otras, se le tenía por una suerte de bruja que vagaba por las noches. Los 
árboles, las fuentes, las cuevas, la luna, los montes y otros astros eran espíritus o moradas de espíritus adorados y consultados por este pueblo.

Un paso importante en la evolución religiosa hebrea fue la monolatría nacional (siglo XII al siglo IX a. C.); es decir, la adoración a un solo Dios con características antropomórficas, defensor del orden del universo. Este era el Dios de Moisés, denominado Yahvé por una construcción de los eruditos (los hebreos solo escribían las consonantes JHWH, porque consideraban que era una ofensa pronunciar el nombre de Dios). Este hecho se aprecia cuando Moisés le preguntó a su Dios su nombre, para poder dar las indicaciones al pueblo, según el texto bíblico, Dios le respondió: “[...] Yo soy el que soy [...]" (Éxodo 3:14, La Biblia).

Si bien los hebreos comenzaron en esta etapa a adorar a un solo Dios, en otros términos, se comenzó a practicar un monoteísmo, esto no significó que en la conciencia colectiva del pueblo hebreo existiera una negación sobre la existencia de otros dioses.

Otra característica relevante de este Dios es que no solamente se preocupaba por sacrificios y ritos, sino también tenía una profunda preocupación por la buena conducta del ser humano y su pureza de corazón. En este contexto es entendible la promulgación de los diez mandamientos que, según el texto bíblico, fueron entregados directamente a Moisés de la mano divina, aunque algunos eruditos consideran que este texto no es del todo fidedigno, porque 
consideran que los diez mandamientos eran anteriores y que lo que se dio en esa época fue su recopilación. Sin embargo, esto no implica un desmérito de que el Dios hebreo posea una característica como la apuntada anteriormente.

Toda esa concepción acerca de Dios, aunque fuera una recopilación, como se aduce, llegó a formar parte del imaginario colectivo de los hebreos, puesto que el imaginario es

[...] el límite entre lo real y lo imaginario, se revela variable, mientras que el territorio atravesado por él permanece al contrario siempre y en todo lugar idéntico, ya que no es otro que el campo entero de la experiencia humana, de lo más colectivamente social y de lo más íntimamente personal [...] (Berenzon, 1999, p. 60).

Conforme pasaron los años, los hebreos comenzaron a no diferenciar entre los distintos dioses y su Dios, de ahí que el relato bíblico ilustre la función preponderante del profeta Elías, que aunque al igual que Moisés, no insistía en la descalificación de otros dioses, sí recalcaba la importancia de volver la mirada hacia el Dios de Moisés, pues este era el único que deberían de adorar los hebreos, porque era el verdadero Dios de la justicia, y quien se preocupaba de manera real por su pueblo.

Posteriormente, otros profetas como Amós, Oseas, Isaías y Miqueas profundizan en la importancia de conocer y adorar a un solo Dios, pero en el 
fondo crearon una revolución religiosa, porque al enfatizar en el aspecto ético de su Dios, dejan de lado la importancia de los ritos y de los sacrificios muy utilizados en la cultura hebrea anterior. De esa manera, comienzan a darle énfasis a los aspectos sociales y políticos, por ejemplo, cuando Miqueas dice “¿Qué pide de ti Yahvé, si no ser justo, y amar la misericordia para andar con tu Dios?" (Miqueas 6:8, La Biblia); porque se debe tener presente que todas las sociedades construyen sus propios mitos, ritos, creencias filosóficas y religiosas que sustentan su visión de mundo y abren espacio a las construcciones de símbolos, así como a la realización de prácticas significantes que producen y reproducen la cultura.

La última gran revolución religiosa hebrea sucedió cuando los hebreos toman conciencia de la existencia de un Dios, porque los demás son dioses falsos, y de que no se debe hacer de su Dios ninguna imagen como antes. Lo anterior se puede apreciar en el texto bíblico que desde el Génesis inicia la idea de un Dios único que paulatinamente se va convirtiendo en la exaltación de un solo Dios que creó el universo, invisible, trascendente e infinitamente superior a todos los seres creados, por lo cual, no puede ser identificado, ni siquiera representado por astros, animales, o seres humanos. De ahí que ninguna imagen de Dios elaborada por mano de hombre pueda considerarse legítima. Esta idea contrasta con religiones como la cananea, en la que existía un vínculo entre la religión y la naturaleza, especialmente, con la fertilidad, como se puede observar con la diosa Abstarté. Contrario a los dioses cananeos, el Dios hebreo no está limitado a los ciclos naturales, sino que Él y 
su voluntad rigen los acontecimientos conforme a sus planes, con un alcance no tribal o nacional, sino universal.

La historia indica que los hebreos no destacaron en campos como la arquitectura, porque las edificaciones más grandes, como el templo construido durante el reinado de Salomón, se hizo con la ayuda fenicia.

\section{Otras} manifestaciones culturales

En el Derecho, su aporte principal se plasma en el código deuteronómico. Se trata de la esencia del libro homónimo, presente en el Antiguo Testamento; no es otra cosa que la síntesis de las disertaciones proféticas de la época. Muchos aspectos presentes en la normativa de este código se encuentran también en otros, por ejemplo, el de Hammurabi, citado anteriormente. Pero, sí se debe indicar que fue el código más cuidadosamente escrito y depurado.

Respecto al desarrollo literario, su aporte más significativo lo contiene el Antiguo Testamento, en el que aparecen textos escritos en prosa y en poesía, asimismo, construcciones al estilo de Confucio y egipcio, como el libro de Eclesiastés y el de Proverbios. Sin embargo, en el caso del libro de Job se cree que está basado en un texto babilónico del mismo nombre y que fue transmitido por la tradición oral. De igual manera, el diluvio universal, descrito en el libro de Génesis recuerda la historia de Gilgamesh, también de origen babilónico. 
En el campo filosófico, se puede decir que los hebreos superaron a las culturas anteriores, pues su constitución religiosa los llevó a un mayor grado de abstracción, al presentar una visión de un Dios universal y no representable de ninguna manera. No obstante, no tuvieron la profundidad de los griegos en sus disertaciones filosóficas.

Siguiendo los fundamentos ideológicos del pueblo hebreo, se tendría que decir que la primera manifestación acerca del tiempo aparece en el libro de

El calendario hebreo

Génesis 1: 14, en donde la creación del sol, la luna y las estrellas fueron imprescindibles para marcar la marcha del tiempo y distinguir entre el día y la noche, establecer las estaciones, los días y los años.

La idea del tiempo en la cultura hebrea fue creada con el propósito de que el hombre se sirviera de este para organizar su vida cotidiana, sus faenas agrícolas, así como la señalización de fechas religiosas y políticas memorables. De esa manera, darle continuidad a la tradición y rescatar su memoria histórica, al igual que todas las culturas.

Las cuatro estaciones en el año hebreo se rigen por el movimiento solar, lo que permite organizar las labores agrícolas, por ejemplo, primavera y verano corresponden a los períodos de siembra y de cosecha.

Como se dijo en apartados anteriores, los hebreos no destacaron en algunos campos, tal es el caso de la astronomía (base fundamental para la elaboración 
de un calendario preciso). Difieren, entonces, a los babilonios y egipcios, quienes sí dominaron este campo; por lo cual, buena parte de los elementos que integran el calendario hebreo provienen de estas culturas.

$\mathrm{Al}$ igual que la religión, en la elaboración de calendarios se indican dos momentos. Uno corresponde a los hebreos nómadas antes de su establecimiento permanente en Palestina, en el que se observa una medición del tiempo distinta, como lo indica el libro del Génesis del Antiguo Testamento, donde, por ejemplo, Matusalén vivió por novecientos sesenta y nueve años, lo cual demuestra que la unidad de tiempo y de año era distinta. No obstante, los datos que se encuentran no son insuficientes para poder reconstruirlo con exactitud. Si calculáramos la edad de Matusalén, tomando como base los ciclos solares, sería de setenta y nueve años aproximadamente.

El otro momento se da cuando los hebreos se establecen en Palestina y se basan en las observaciones de los ciclos lunares heredadas de la cultura babilónica y egipcia. Plantean que el mes lunar es el tiempo que media entre una luna nueva y la siguiente, esto los conduce a determinar que algunos meses están conformados por treinta días, y otros por veinte y nueve y 1/2 días. Por lo tanto, se puede decir que el calendario hebreo es lunisolar, porque, como se mencionó anteriormente, para calcular el inicio de cada estación recurren a las observaciones astronómicas de las culturas anteriores, que se 
basaban en la posición de las estrellas con respecto al sol en cada momento del año.

El día de inicio de un nuevo mes lo denominaron Rosh Jódesh que significa cabeza de mes y, si el mes anterior estaba compuesto por treinta días, el Rosh Jódesh era celebrado por dos días, lo cual dio lugar a festejos especiales en el pueblo de Israel.

Siguiendo los movimientos de la luna, el año hebreo se conforma por doce meses que suman trescientos cincuenta y cuatro días y $1 \frac{1}{2}$, lo que indica que el año es once días más corto que el año solar compuesto por trescientos sesenta y cinco días y fracción, como lo plantea el calendario gregoriano que es solar. De ahí que sea necesario que cada tres años, se agregue un mes al calendario hebreo, este recibe el nombre de Adar y como el último mes del año se denomina Adar, cuando el calendario hebreo tiene que ser arreglado, por lo explicado anteriormente, encontramos dos meses con el mismo nombre.

Los meses del calendario, antiguamente, se denominaron por su orden numérico. Estos comenzaban en la primavera (boreal), el mes primero se llamó Nisán; este es muy significativo para los hebreos, porque fue en este mes cuando el pueblo de Israel salió de Egipto (por eso se considera el primer mes de año). La influencia babilónica también se refleja en todos los nombres de los meses. 
Actualmente, el orden cronológico de los meses es el siguiente: Nisán (marzo-abril), Iyar (abril-mayo), Sivan (mayo-junio), Tamuz (junio, julio), Av (julio-agosto), Elul (agosto-setiembre), Tishrei (setiembre u octubre), Jeshvan o Marjeshvan (octubre o noviembre), Kislev (noviembre o diciembre), Tevet (diciembre-enero), Shevath (enero-febrero), Adar (febreromarzo). Sin embargo, en el Antiguo Testamento, se mencionan algunos meses, no todos, pero sí se especifica claramente el mes que da inicio al año, "Este mes os será principio de los meses; para vosotros será este el primero en los meses del año" (Éxodo 12:2, La Biblia). La tradición judía actual ha establecido, a pesar de la cita bíblica antes mencionada, que el inicio del año es el mes de Tishrie (setiembre-octubre), porque según sus cálculos fue el mes de la creación del mundo y la tradición ha prevalecido; por ello, se puede afirmar que un criterio universalista se impuso al criterio nacionalista planteado por el texto bíblico.

El calendario hebreo es eminentemente religioso y los días de las semanas se nombran por ordinales, comenzando por el día domingo, para ajustarse a los días de la creación del mundo, tal como lo explica el libro del Génesis del Antiguo Testamento. Por eso, los primeros seis días son laborales y el último o séptimo, denominado Shabbath, es día de reposo (como su nombre lo indica), para dar continuidad a lo escrito en el Génesis. También el sábado se consideraba día de descanso, para recordar la esclavitud que vivió el pueblo en Egipto. 
Dentro de ese contexto religioso, los hebreos le otorgaron importancia a ciertas fechas que son indispensables para su memoria histórica. Por ejemplo, el mes Nisán conmemora la salida de Egipto; y el mes Elul es cuando se debe de pagar el diezmo que es, por supuesto, un elemento vital en la religión judía, porque servía para el sostenimiento de los sacerdotes. En el mes Tishrei se celebra la creación del mundo, además, servía como base para calcular el descanso de la tierra, para prepararla con el fin de iniciar los cultivos; de igual forma, durante este mes se da el jubileo, que se celebraba cada cincuenta años, este consistía en la prescripción de las deudas y la liberación de los esclavos.

La celebración de la Pascua judía tiene su origen en el período patriarcal, cuando los clanes nómadas atravesaron el desierto en busca de pastos para sus rebaños. Antes de la partida, ofrecían un sacrificio a su dios protector para que su rebaño fuese fecundo, para tener seguridad en el camino y protección contra las enfermedades, pestes, tormentas y muertes. El sacrificio consistía en separar un animal recién nacido que no presentara ninguna anormalidad y ofrendarlo al atardecer. La carne se asaba al fuego y todos se congregaban para una cena en comunión entre ellos y su divinidad.

A lo largo del tiempo, este ritual se transforma, especialmente después de la salida de la esclavitud y cuando el pueblo hebreo emprende camino hacia 
Palestina; se establece su fecha en primavera. En el texto bíblico,

Deuteromonio 16:1-8, se señala que antes del destierro a Babilonia, en el año 587 antes de Cristo, se centraliza la celebración de la Pascua en Jerusalén. Así, esta celebración expresaba su objetivo fundamental que era honrar a un solo Dios, un solo pueblo y un único santuario.

La celebración de la Pascua fue asumida por la Iglesia Católica y en el calendario gregoriano se establece que se debe efectuar de acuerdo con la fecha que coincida con la primavera hebrea.

La cultura hebrea es una cultura con más tradición nómada que sedentaria. Por este motivo, quizás, los hebreos no realizaron observaciones astronómicas que les permitieran desarrollar un calendario solar; por eso, recurrieron a datos astronómicos de las grandes culturas sedentarias de Babilonia y Egipto, que no solo observaron el firmamento, sino también, relacionaron dichas observaciones con fenómenos terrestres como la crecida de los ríos y la llegada de la estación seca, lo que les permitió su calendarización.

El hecho de ser nómadas, por mucho tiempo, tampoco les permitió desarrollar significativamente otras áreas culturales, ni dejar un legado importante a la humanidad, como sí lo hicieron otras civilizaciones. Sin embargo, el gran aporte de ellos fue en el campo religioso, al señalar la existencia de único Dios, intangible, invisible e irrepresentable que, en un principio, fue únicamente para la cultura hebrea y luego tomó carácter casi universal, 
porque a partir de los textos del Antiguo Testamento, surgirá una figura central en el Nuevo Testamento, que aunque no es aceptado por los hebreos como Dios, sí será de mucha importancia para el desarrollo del cristianismo en Occidente.

El legado hebreo en el campo religioso toma mayor importancia a partir del nacimiento de Jesucristo, a tal punto que este acontecimiento se convierte en parámetro para medir el tiempo en las culturas occidentales, porque los hechos históricos se establecen antes de y después de Cristo. Además, la muerte de Jesucristo coincidente con la festividad pascual hebrea, lo que ha repercutido en la confección de los calendarios occidentales, porque se hizo necesario hacer coincidir la celebración de la Semana Santa con la primavera hebrea; en esta época se conmemora la salida del pueblo hebreo de Egipto.

Otro legado importante del pueblo hebreo para la humanidad fue la creación de un Dios monoteísta, universal y ético. 
Assmann, Jann (2003). Moisés el egipcio. Madrid, España: Grupo Anaya S.A.

Artigas, M (2005). Segunda Antología Sefardí: continuidad cultural (1600-

Referencias bibliográficas 1730). Madrid, España: Editorial Verbm, S.L.

Ausejo, Serafín (2004). Diccionario de la Biblia. Barcelona, España:

Editorial Herder.

Berenzon, Boris (1999). Historia es inconsciente. México: Ediciones, Colegio San Luis.

Bentor, Amnon (2004). La arqueología del Antiguo Israel. Madrid: Ediciones cristiandad S.A.

Del Olmo, Lete (2010). Origen y persistencia del judaísmo. España: Editorial Verbo Divino.

Finkelstein, Israel y otros (2003). La Biblia desenterrada. Una nueva visión arqueológica del antiguo Israel y los orígenes de los textos sagrados. Madrid, España: Ediciones Siglo XXI.

García, Félix (2003). El Pentateuco. Introducción a la lectura de los cinco primeros libros de la Biblia. Navarra, España: Editorial Verbo divino.

Lara, Federico (1986). Código de Hammurabi; estudio preliminar, traducción y comentarios. Madrid, España: Editorial Técnos. 
León, Abram (1943). Historia de los judíos. Santiago de Chile: Ediciones Ercilla.

Levi, Leo y otros (1982). Israel. El pueblo judío, su historia y sus tradiciones. Jerusalén: Departamento de Educación y cultura religiosa para la Diáspora de la Organización Sionista Mundial.

Lods, Adolphe (1940). La religion de Israel. Buenos Aires, Argentina: Editorial Rodríguez Giles.

Martínez, José (1987). Hermeneútica Bíblica. Barcelona, España: Editorial Clie.

Mickelsen, A (1986). La interpretación de la Biblia. Barcelona, España: Editorial Herder.

McNalls, Burns, Edward. (1970). Civilizaciones de Occidente. Buenos Aires, Argentina: Ediciones Peuser.

Pixley, J. (1983). Exodo una lectura evangélica popular. México. Editorial Casa Unida de Publicaciones S.A.

Reeves, Nicholas (2002). Akhenatón falso profeta de Egipto. Madrid. Grupo Anaya. 
Reingold, Edward y otros (2001). Cálculos calendáricos. Inglaterra. Ediciones Milenium. Cambridge University Press.

Sacchi, Paolo (2004). Historia del judaísmo en la época del Segundo Templo: Israel. Perú: Editorial Trotta Pontificia Universidad Católica.

Santa Biblia. Versión revisada, (1997). Tennessee, Estados Unidos. Broadman and Holman Publishers.

Wright, Ernest G (2002). Arqueología Bíblica. Madrid. Ediciones Cristiandad.

Wright, Georges y otros (1979). Atlas histórico Westminster de la Biblia. El Paso, Texas, Estados Unidos. Casa Bautista de Publicaciones. 